\title{
A Linac Afterburner to Supercharge the Fermilab Booster
}

\author{
C. Ankenbrandt, J. MacLachlan, M. Popovic, R. P. Johnson
}

Fermi National Accelerator Laboratory

\begin{abstract}
A Linac Afterburner is proposed to raise the energy of the beam injected into the Fermilab Booster from 400 $\mathrm{MeV}$ to about $600 \mathrm{MeV}$, thereby alleviating the longitudinal and transverse space-charge effects at low energy that currently limit its performance. The primary motivation is to increase the integrated luminosity of the Tevatron Collider in Run II, but other future programs would also reap substantial benefits. The estimated cost is $\$ 23 \mathrm{M}$.
\end{abstract}

\section{INTRODUCTION}

The Fermilab Linac Upgrade Project, completed in 1993 at a total cost of $\$ 31 \mathrm{M}$, increased the linac energy from 200 to $400 \mathrm{MeV}$. The Afterburner proposal emulates that successful project in several respects. The same kind of warm copper $805-\mathrm{MHz}$ side-coupled cavities are used to accelerate the beam. Once again, existing enclosures and buildings are used to house the accelerator components and their supporting equipment. The installation and commissioning are planned to minimize their interference with Run II. Emulating the previous successful project allows a quick start and a rapid completion of the Afterburner Project. The luminosity of the Tevatron Collider increased by a factor of about 2.5 after the Linac Upgrade Project; the Afterburner is expected to provide a corresponding luminosity enhancement in Run II.

\section{THE BOOSTER AND THE AFTERBURNER}

When the Booster runs at $5 \cdot 10^{12}$ protons per cycle, as called for in the Main Injector era, several mechanisms of emittance growth and beam loss are evident. In the first few milliseconds after injection, transverse space-charge effects cause rapid emittance growth accompanied by substantial $(-30 \%)$ beam losses. Meanwhile, longitudinal space-charge forces distort the potential well created by the rf focusing force, causing longitudinal emittance growth and failure to capture part $(\sim 10 \%)$ of the beam. Of course that uncaptured beam is swept radially inward out of the aperture as soon as the magnetic field ramps up significantly. These two low-energy space-charge effects are the dominant beam-loss mechanisms in normal operation, but they are not the whole story.
Although aggressive pursuit of measures to control higher-energy losses is certainly necessary to the success of the near-future physics program, only modest intensity gains are likely to result, because the higher-energy losses amount to less than ten percent of the beam.

The Booster is currently the bottleneck preventing higher-intensity operation of the whole facility, because both the upstream and downstream machines, the Linac [1] and the Main Injector/Tevatron complex, can handle higher intensities than the Booster.

\section{AFTERBURNER TECHNICAL DESIGN CONCEPTS}

To accelerate the beam between the Linac and the Booster, accelerating structures like those in the downstream end of the Linac will be installed along the outer wall of the Booster tunnel. (Putting a curved linac in the Booster tunnel was previously suggested in another context.[2]) The Afterburner is preceded by a longitudinal matching section and followed by a debunching section. The existing 400-MeV linac and beam transfer line to the Booster are left essentially untouched up to $\mathrm{MH} 2$, a horizontal bending magnet near the outer tunnel wall that currently bends the beam from the linac across the aisle toward the Booster injection system.

\section{A. The Afterburner}

The Afterburner is based on the $805 \mathrm{MHz}$ warm copper side-coupled cavities installed at the downstream end of the existing linac during the Linac Upgrade Project [3]. There are several advantages to that choice. The technology is well understood and the costs well known, so no R\&D is necessary prior to writing a definitive proposal. The transverse size of the side-coupled cavities is small enough to fit comfortably in the tunnel. Spare parts, including 
several 12 MW klystrons, exist and can be shared with the present system. Most importantly, the present system works very well, reliably producing highquality beam with low beam losses. The decision to employ that technology allows the quick start and rapid completion that are essential in order to benefit Run II.

The Booster itself consists of 24 periods, with each period containing a long straight section, two combined-function magnets, a short straight section, and two more combined-function magnets. Figure 1 shows the layout of the Booster in its tunnel on the south side, where the Afterburner is to be installed. The length of the outer tunnel wall is 20.5 meters per period. The shape of the Afterburner and its transfer lines is regular and symmetrical, like the Booster and most of its tunnel. There are 7.5 degree bend centers opposite the middle of the short and long straight sections, separated by 10.25 meters. The 10.25 meter distance between bend centers is sufficient to accommodate one accelerating module along with the required bending and focusing magnets. In the present design there are actually five small magnets at the bend centers: a quadrupole triplet with two 3.75 degree dipoles interspersed. The dipoles are 0.5 meters long to reduce field stripping to negligible proportions.

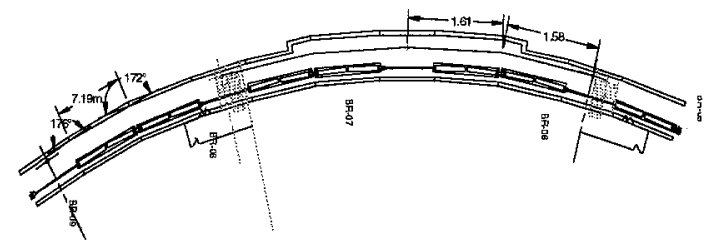

\section{FIGURE 1.}

The accelerating structure has five modules. Each module consists of four sections joined by bridge couplers to accommodate quadrupole focusing magnets. Each section contains twelve accelerating cavities. The choice of twelve cavities per section, rather than sixteen as in the existing linac, makes the modules short enough so that the Afterburner can match the shape of the outer tunnel wall. The rest of the parameters are the same as in the existing linac.

The maximal accelerating field is limited by the requirement that the electric field is less than 1.4 times the Kilpatrick value. The shunt impedance of the structure is taken to be $80 \%$ of the value calculated by Superfish; the same criterion was used in the design of the $400 \mathrm{MeV}$ energy upgrade. The transit-time factor, maximal field, and shunt impedance are extrapolated from the Superfish calculation for the Linac Upgrade Project for beta in the region from $0.57<\beta<0.71$.
Figure 2, taken from Trace3D output, shows the phase-space ellipses in the transverse and longitudinal dimensions. In the upper left corner are input beam ellipses with alphas and betas for three planes. The upper right corner shows the same beam parameters at the end of the Afterburner. The lower portion of the graph shows beam envelopes and accelerator elements. The displayed envelopes are the square root of five times their respective rms values. The two upper traces are the horizontal envelope and bunch length and the lower trace is the vertical envelope. The quad settings are adjusted to keep the beam envelope small along the linac. The full scale of $15 \mathrm{~mm}$ corresponds to the radius of the physical aperture.

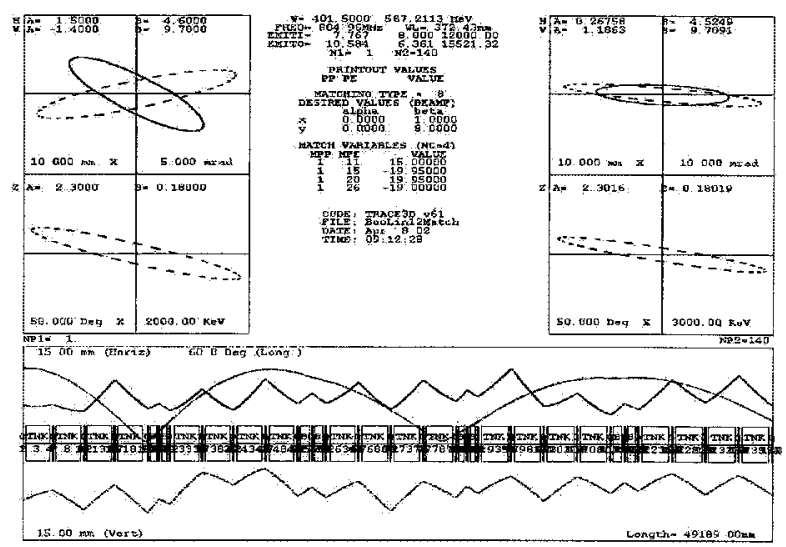

\section{FIGURE 2.}

It is interesting to note the similarity of Figure 2 with the corresponding Figure 15 of the Fermilab Linac Upgrade Conceptual Design Report [Ref]. In particular the beam envelopes in both cases are contained within about half of the physical aperture. That shows that the dispersion associated with the required bends is not a problem. The momentum spread contributes only a few $\mathrm{mm}$ of beam size in quadrature with the transverse beam sizes. The transverse sizes shrink adiabatically from their values at $116 \mathrm{MeV}$ while the physical apertures remain the same size.

\section{B. Longitudinal Matching}

The Afterburner requires a buncher to match into its side-coupled structure (SCS) at the upstream end and a debuncher at the downstream end to reduce the momentum spread for injection into the Booster. (Transverse matching is of course also required, but it is not discussed here because it is routine.) The bunching system makes use of the existing $400 \mathrm{MeV}$ debuncher but requires an additional bunching tank closer to the Afterburner. 
The existing $805 \mathrm{MHz}$ debuncher is located about $30 \mathrm{~m}$ downstream of the end of the $400 \mathrm{MeV}$ SCS linac where the beam bunches have sheared to span about $\mathbf{8 0}$ degrees of $\mathrm{rf}$ phase. This is a good spread for rebunching with good linearity using a modest $\mathrm{rf}$ system. However, it is much too far upstream to permit the sharp focus (small longitudinal beta function) required to match to the Afterburner. This design includes a new buncher structure located about $25 \mathrm{~m}$ upstream of the Afterburner. To reduce the power required for the buncher, a twelve-cell SCS is used. It requires about $480 \mathrm{~kW}$ of rf power, which is not low enough to use the same kind of small TV klystron that powers the first buncher, so a shorter structure powered by one of the $12 \mathrm{MW}$ klystrons might be preferred. The existing four-cell debuncher is excited to $2 \mathrm{MV} / \mathrm{m}$ to keep the bunch width below one hundred degrees. The new buncher is excited to an effective gradient of $1.7 \mathrm{MV} / \mathrm{m}$ so that the bunches entering the Afterburner have the same distribution in longitudinal phase space as the ones exiting the 400 $\mathrm{MeV}$ linac: 12 degrees full width and $2.6 \mathrm{MeV}$ full energy spread.

The $600 \mathrm{MeV}$ debuncher serves exactly the same purpose as the existing debuncher in the present transfer line between the $400 \mathrm{MeV}$ linac and the Booster, but substantially more power is needed. It can be located $40 \mathrm{~m}$ downstream of the Afterburner and run at exactly the same gradient as the buncher. The energy spread of the $600 \mathrm{MeV}$ beam is reduced from $2.8 \mathrm{MeV}$ full width to $0.54 \mathrm{MeV}$ at the Booster, corresponding to a momentum spread of $0.03 \%$.

\section{Injection into the Booster}

A new injection system to be located at Long Straight Section 11 is a part of this proposal. The existing injection system will be left where it is at Long Straight Section 1 so that it can continue to be used for injection of $400-\mathrm{MeV}$ beam until the commissioning of the Afterburner is complete. Temporarily having dual injection systems will reduce the interruption to Run II, and a new injection system can incorporate the latest design ideas. Besides, the existing injection system probably would not work at $600 \mathrm{MeV}$ anyway.

Figure 3 shows the layout of the new $\mathrm{H}^{-}$injection system. The design borrows ideas from that of the injection system for the Proton Driver[4], but unlike that system it is local, that is, confined to a single straight section with no lattice elements interspersed among injection components.

Injecting a high-energy beam in a limited space is a design challenge because the field strength $\mathrm{B}$ of the magnets that transport the incoming ion beam must be limited; they must satisfy the constraint $\mathrm{B}<0.7$ Tesla/p $(\mathrm{GeV} / \mathrm{c})$ in order to reduce the probability of fieldstripping to about $10^{-3}$. The parameter choices used in Figure FFF-INJ satisfy that constraint and comfortably accommodate $600 \mathrm{MeV}$ beam in the available space. If necessary considerably higher incoming kinetic energies could be injected using this kind of design.

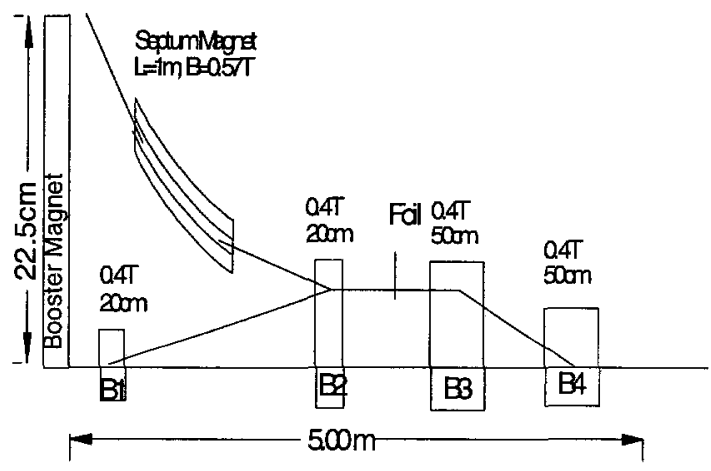

FIGURE 3.

\section{SUMMARY AND CONCLUSIONS}

A Linac Afterburner is proposed to supercharge the Fermilab Booster by raising its injection energy, thereby alleviating the transverse and longitudinal space charge effects that currently limit its performance. The Booster is the bottleneck in the whole Fermilab accelerator complex because all the other machines can handle higher intensity beams. Considerable leverage is involved because a modest improvement upstream of the Booster would benefit all components of the Fermilab experimental high energy physics program. Increasing the brightness and intensity of the Booster and Main Injector beams would be quite valuable to the US HEP program. However, the primary motivation for this proposal is to increase the integrated luminosity for Run II of the Tevatron Collider. CERN's remarkable effort to discover the Higgs in the last LEP run was impressive; now Fermilab must pick up the torch. A Linac Afterburner is the logical Fermilab response to the mandate to maximize the discovery potential of Run II. This document has demonstrated the feasibility of the Afterburner Project. The nature and scope of the project would be much like that of the highly successful Linac Upgrade Project. A quick start is possible because no $R \& D$ is necessary; we already know everything we need to know to write a credible proposal to the Department of Energy. A rapid completion is possible because the cost and scope are modest and because existing structures can be used to house the accelerating components and supporting 
equipment. Can Fermilab afford not to pursue these opportunities vigorously?

\section{REFERENCES}

1. LINAC Staff (M. Popovic for the collaboration), PRESENT, NEAR FUTURE AND FUTURE PERFORMANCE OF THE FERMILAB LINAC, FERMILAB-PUB-96-046, Mar 1996

2. M. Popovic, A. Moretti, R. Noble, and C.W. Schmidt, 1-GeV Linac Upgrade Study at Fermilab, 1998 Linac Conf

3. http://wwwlib. fnal.gov/archive/linac/FERMILABLU-ConceptualDesign.pdf

4. W. Chou, C. Ankenbrandt, and E. Malamud, Eds., The Proton Driver Design Study, FERMILABTM-2136, December 2000. 